[25] V. V. Veeravalli, T. Başar, and H. V. Poor, "Decentralized sequential detection with a fusion center performing the sequential test," IEEE Trans. Inform. Theory, vol. 39, no. 2, pp. 433-442, Mar. 1993.

[26] V. V. Veeravalli, T. Başar, and H. V. Poor, "Decentralized sequential detection with sensors performing sequential tests," Math.. Control, Signals, Syst., vol. 7, no. 4, pp. 292-305, 1994.

[27] P. Willett, P. F. Swaszek, and R. S. Blum, "The good, bad, and ugly: Distributed detection of a known signal in dependent gaussian noise," IEEE Trans. Signal Process., vol. 48, no. 12, pp. 3266-3279, Dec. 2000.

[28] P. Varaiya and J. Walrand, "On delayed sharing patterns," IEEE Trans. Autom. Control, vol. AC-23, no. 3, pp. 443-445, Mar. 1978.

[29] A. Gupta and R. Sekar, "An approach for detecting self-propagating email using anomaly detection," in Recent Advances in Intrusion Detection, ser. Lecture Notes in Computer Science, G. Vigna, E. Jonsson, and C. Kruegel, Eds. Berlin, Germany: Springer-Verlag, 2003, pp. $55-72$.

\section{Quadratic Stability and Singular SISO Switching Systems}

\author{
Robert Shorten, Martin Corless, Kai Wulff, Steffi Klinge, and \\ Richard Middleton
}

\begin{abstract}
In this note, we consider the problem of determining necessary and sufficient conditions for the existence of a common quadratic Lyapunov function for a pair of stable linear time-invariant systems whose system matrices are of the form $A, A-g h^{T}$, and where one of the matrices is singular. A necessary and sufficient condition for the existence of such a function is given in terms of the spectrum of the product $A\left(A-g h^{T}\right)$. The technical note also contains a spectral characterization of strictly positive real transfer functions which are strictly proper. Examples are presented to illustrate our results.
\end{abstract}

Index Terms-LTI systems.

\section{INTRODUCTION}

Consider a switching system described by

$$
\dot{x}=\left[A-\sigma(t, x) g h^{T}\right] x
$$

where the state $x(t)$ and $g, h$ are real vectors, $A$ is a real square matrix, and the scalar switching function $\sigma$ satisfies

$$
0 \leq \sigma(t, x) \leq 1
$$

Suppose $A$ is a Hurwitz matrix, that is, all its eigenvalues have negative real parts; then the system corresponding to $\sigma(t, x) \equiv 0$, that is, $\dot{x}=A x$, is globally asymptotically stable about the origin of the state space. Suppose also that all the eigenvalues of $A-g h^{T}$ have negative real parts except for a single eigenvalue at zero. Then the system corresponding to $\sigma(t, x) \equiv 1$, that is, $\dot{x}=\left(A-g h^{T}\right) x$, is stable

Manuscript received July 16, 2008; revised December 01, 2008. First published October 16, 2009; current version published November 04, 2009. This work was supported by Science Foundation Ireland under Grants 07/IW.1/1901 and 07/RPR/I177. Recommended by Associate Editor D. Liberzon.

R. Shorten, K. Wulff, S. Klinge, and R. Middleton are with the Hamilton Institute, NUI Maynooth, Maynooth, Ireland (e-mail: robert.shorten@nuim.ie; richard.middleton@nuim.ie; kai.wulff@tu-ilmenau.de; mail@steffi-klinge.de).

M. Corless is with the School of Aeronautics and Astronautics, Purdue University, West Lafayette, IN 47907 USA (e-mail: corless@ purdue.edu).

Digital Object Identifier 10.1109/TAC.2009.2031586 (but not asymptotically stable) about the origin and all its solutions are bounded. Consider now the switching system (1) with any switching function $\sigma$ which satisfies the constraint (2). One can show that this system is stable about the origin and all solutions are bounded if there is a real symmetric positive definite matrix $P$ satisfying the following two Lyapunov matrix inequalities:

$$
\begin{aligned}
A^{T} P+P A & <0 \\
\left(A-g h^{T}\right)^{T} P+P\left(A-g h^{T}\right) & \leq 0 .
\end{aligned}
$$

This can be justified as follows. As a candidate Lyapunov function for system (1)-(2) consider the quadratic function $V(x)=x^{T} P x$. Then, along any solution $x(\cdot)$ of the system we have

$$
\dot{V}=2 x^{T} P A x-2 \sigma(t, x) x^{T} P g h^{T} x .
$$

Inequalities (3) imply that $\dot{V} \leq 0$ when $\sigma(t, x)$ equals 0 or 1 . Noting that $\dot{V}$ depends in a linear affine fashion on $\sigma(t, x)$, it now follows that $\dot{V} \leq 0$ for any $\sigma(t, x)$ in the interval $[0,1]$. Since $V$ is a positive definite function, standard Lyapunov theory guarantees the claimed stability/ boundedness properties.

Stability problems involving systems described by (1)-(2) arise in a variety of applications; for example, in applications where integrators are switched in and out of feedback loops to achieve certain performance objectives [1]-[4].

We refer to a matrix $P=P^{T}>0$ satisfying (3) as a common Lyapunov matrix for $A$ and $A-g h^{T}$; the corresponding Lyapunov function $V(x)=x^{T} P x$ is referred to as a common quadratic Lyapunov function. Assuming that $(A, g)$ is controllable and $(A, h)$ is observable, we show that the following simple condition is both a necessary and sufficient condition for the existence of a common Lyapunov matrix $P$.

The matrix product $A\left(A-g h^{T}\right)$ has no negative real eigenvalues and exactly one zero eigenvalue.

Comment 1: We have assumed that $A-g h^{T}$ has an eigenvalue at zero. One can relax this to the requirement that $A-g h^{T}$ is marginally stable but not Hurwitz. To see this, one can show that, in order for the Lyapunov inequalities (3) to be satisfied, $A-g h^{T}$ can have only one eigenvalue at zero and no other eigenvalues on the imaginary axis; see the Appendix (First note). One can also show that if $A-g h^{T}$ is marginally stable, $A$ is Hurwitz and the conditions on the eigenvalues of $A\left(A-g h^{T}\right)$ hold, then $A-g h^{T}$ can have only one eigenvalue at zero and no other eigenvalues on the imaginary axis; see the Appendix (Second Note).

In the previous literature, the results most closely related to this technical note are contained in [5], [6]. Both of these papers consider quadratic stability of switched systems where both constituent matrices $A_{1}, A_{2}$ are Hurwitz stable. In the first paper, conditions are given for the existence of a CQLF for a pair of second order LTI systems in terms of the spectrum of $A_{1} A_{2}$ and $A_{1} A_{2}^{-1}$, and in the second paper, conditions are given for a pair of matrices of arbitrary order, but whose rank difference is one.

Before proceeding it is useful to note that the CQLF existence problem for pairs of LTI systems, one of which is marginally stable, is substantially more difficult than the equivalent problem when both LTI systems are Hurwitz stable. To see that results do not immediately follow from one problem to another, consider again the problem treated in the second of the aforementioned papers [6]. Here the authors consider two Hurwitz matrices $A_{1}, A_{2}$ in companion form and show that there exists a real matrix $P=P^{T}>0$ satisfying $P A_{i}+A_{i}^{T} P<0$ for $i=1,2$ if and only if the matrix product $A_{1} A_{2}$ has no negative real eigenvalues. One may be tempted to conclude that one can readily obtain the results of the present paper using the result 
in [6] by using the following reasoning. For $\epsilon>0$ sufficiently small, let $A_{2}(\epsilon)$ be a perturbation of $A-g h^{T}$ such that $A_{2}(\epsilon)$ is Hurwitz and the product $A A_{2}(\epsilon)$ has no negative real eigenvalues. Then, for each $\epsilon>0$ sufficiently small [6] tells us that there is a matrix $P(\epsilon)$ satisfying $P(\epsilon) A+A^{T} P(\epsilon)<0$ and $P(\epsilon) A_{2}(\epsilon)+A_{2}(\epsilon)^{T} P(\epsilon)<0$. Now consider limits as $\epsilon$ goes to zero. The following counterexample illustrates why this proof technique will not work.

1) Example: Consider

$$
\begin{aligned}
A & =\left[\begin{array}{cc}
0 & 1 \\
-1 & -1
\end{array}\right] \quad A-g h^{T}=\left[\begin{array}{cc}
0 & 1 \\
0 & -1
\end{array}\right] \\
A_{2}(\epsilon) & =\left[\begin{array}{cc}
0 & 1 \\
-\epsilon & -1
\end{array}\right] .
\end{aligned}
$$

Here, $g=\left[\begin{array}{ll}0 & 1\end{array}\right]^{T}, h=\left[\begin{array}{ll}-1 & 0\end{array}\right]^{T}$ and one can readily verify that $(A, g)$ is controllable and $(A, h)$ is observable. Clearly $A$ and $A_{2}(\epsilon)$ are Hurwitz for all $\epsilon>0$ and $A_{2}(0)=A-g h^{T}$. The characteristic polynomial of the matrix $A A_{2}(\epsilon)$ is given by $d(s)=s^{2}+\epsilon s+\epsilon$. For $0<\epsilon<4$, this polynomial has complex roots. Hence, for this range of $\epsilon, A_{1} A_{2}(\epsilon)$ has no real negative eigenvalues and it follows from [6] that there is a matrix $P(\epsilon)=P(\epsilon)^{T}>0$ satisfying $P(\epsilon) A+A^{T} P(\epsilon)<$ 0 and $P(\epsilon) A_{2}(\epsilon)+A_{2}(\epsilon)^{T} P(\epsilon)<0$. However the matrix product $A\left(A-g h^{T}\right)=A A_{2}(0)$ has a repeated eigenvalue at zero. Hence, using the result of this technical note, there does not exist a matrix $P=P^{T}>0$ satisfying (3).

In the next section, we present some results on positive real transfer functions which are useful in the development of the main result. In particular, Theorem 2.1 provides a simple spectral characterization of strictly positive real transfer functions. We believe this is a useful result on its own and not just for the purposes of obtaining the main result. Section III develops the main result of this technical note. To achieve this we also need the Kalman-Yacubovich-Popov lemma for proper SISO systems that is found in most textbooks; see the book by Boyd [7] or Khalil [8]. Throughout, known results are quoted without proof whereas new results are given with full proofs.

\section{SPeCtRal Characterizations OF STRICT POSITIVE REALNESS}

Before obtaining our main result, we obtain some preliminary results on strictly positive real (SPR) transfer functions. In everything that follows, $A$ is a real $n \times n$ matrix and $b, c$ are real $n$-vectors.

Recall that a scalar transfer function $H$ is strictly positive real (SPR) if there exists a scalar $\alpha>0$ such that $H$ is analytic in a region of the complex plane which includes those $s$ for which $\operatorname{Re}(s) \geq-\alpha$ and

$$
H(j \omega-\alpha)+H(j \omega-\alpha)^{*} \geq 0 \quad \forall \quad \omega \in \mathbb{R} .
$$

We say $H$ is regular if $H(j \omega)+H(j \omega)^{*}$ is not identically zero for all $\omega \in \mathbb{R}$. For convenience, we will include regularity as a requirement for SPR.

The following standard result provides a more convenient characterization of SPR. It eliminates $\alpha$.

Lemma 2.1: [8]: Suppose $A$ is Hurwitz. Then the transfer function $H(s)=c^{T}(s I-A)^{-1} b$ is SPR if and only if

$$
H(j \omega)+H(j \omega)^{*}>0 \quad \forall \omega \in \mathbb{R}
$$

$$
\lim _{\omega \rightarrow \infty} \omega^{2}\left[H(j \omega)+H(j \omega)^{*}\right]>0
$$

In checking SPR of a system it is sometimes more convenient to check SPR of a system which is equivalent (from an SPR viewpoint) to the original system. The following lemma provides such an equivalent system and is useful for generating some of the results of this technical note. This system is simply obtained by replacing $A$ with $A^{-1}$.

Lemma 2.2: The transfer function $H(s)=c^{T}(s I-A)^{-1} b$ is SPR if and only if $H_{I}(s)=c^{T}\left(s I-A^{-1}\right)^{-1} b$ is SPR.

Proof: Suppose $H$ is SPR. The identity $\left(s I-A^{-1}\right)^{-1}=s^{-1} I-$ $s^{-2}\left(s^{-1} I-A\right)^{-1}$ implies that

$$
H_{I}(s)=s^{-1} c^{T} b-s^{-2} H\left(s^{-1}\right)
$$

hence we get the equation shown at the bottom of the page. Considering limits as $\omega \rightarrow 0$

$$
H_{I}(0)+H_{I}(0)^{*}=\lim _{\tilde{\omega} \rightarrow \infty} \tilde{\omega}^{2}\left[H(j \tilde{\omega})+H(j \tilde{\omega})^{*}\right]>0 .
$$

Finally, we note that

$$
\lim _{\omega \rightarrow \infty} \omega^{2}\left[H_{I}(j \omega)+H_{I}(j \omega)^{*}\right]=H(0)+H(0)^{*}>0 .
$$

The core of our main result is based on a spectral condition for strict positive realness of strictly proper transfer functions; this is related to corresponding results in [9] for transfer functions which are proper but not strictly proper. This result makes use of the following lemma.

Lemma 2.3: [10]-[12]: Let $H(s)=d+c^{T}(s I-A)^{-1} b$ where $A$ is invertible. Then, $H\left(s^{-1}\right)=\bar{d}+\bar{c}^{T}(s I-\bar{A})^{-1} \bar{b}$ with $\bar{A}=A^{-1}$, $\bar{b}=-A^{-1} b, \bar{c}^{T}=c^{T} A^{-1}$ and $\bar{d}=d-c^{T} A^{-1} b$.

1) Comment 2: Note that when $H$ is SPR we must have $\bar{d}>0$. This follows from the fact that $\bar{d}=H(0)$ and $H(0)+H(0)^{*}>0$ since $H$ is SPR.

Now we give the aforementioned spectral characterization of strict positive realness.

Theorem 2.1: Suppose $A$ is Hurwitz. Then, the following statements are equivalent.

(a) The transfer function $H(s)=c^{T}(s I-A)^{-1} b$ is SPR.

(b) $c^{T} A^{-1} b<0$ and the matrix product $A^{-1}\left(A^{-1}-\right.$ $\left.A^{-1} b c^{T} A^{-1} / c^{T} A^{-1} b\right)$ has no negative real eigenvalues and exactly one zero eigenvalue.

(c) $c^{T} A b<0$ and the matrix product $A\left(A-A b c^{T} A / c^{T} A b\right)$ has no negative real eigenvalues and exactly one zero eigenvalue.

Proof: In what follows it is convenient to work with $H\left(s^{-1}\right)$ as in Lemma 2.3. In particular, the conditions for SPR of $H$ may be restated in terms of the transfer function $H\left(s^{-1}\right)$. Specifically, conditions (5) and (6) for SPR of $H$ are equivalent to

$$
\begin{aligned}
\lim _{\omega \rightarrow \infty} H\left(-j \omega^{-1}\right)+H\left(-j \omega^{-1}\right)^{*} & >0 \\
H\left(-j \omega^{-1}\right)+H\left(-j \omega^{-1}\right)^{*} & >0 \text { for } \omega \neq 0 \\
\lim _{\omega \rightarrow 0} \frac{1}{\omega^{2}}\left[H\left(-j \omega^{-1}\right)+H\left(-j \omega^{-1}\right)^{*}\right] & >0 .
\end{aligned}
$$

Condition (8) is equivalent to $c^{T} A^{-1} b<0$. Now consider conditions (9) and (10). Since $A$ is invertible, Lemma 2.3 tells us that

$$
H\left(-j \omega^{-1}\right)=\bar{d}+\bar{c}^{T}(j \omega I-\bar{A})^{-1} \bar{b}
$$

$$
H_{I}(j \omega)+H_{I}(j \omega)^{*}=\omega^{-2}\left[H\left(-j \omega^{-1}\right)+H\left(-j \omega^{-1}\right)^{*}\right]>0 \quad \forall \quad \omega \neq 0 .
$$


with $\bar{A}, \bar{b}, \bar{c}, \bar{d}$ defined in Lemma 2.3. Using the results in [13], we have $\bar{c}^{T}(j \omega I-\bar{A})^{-1} \bar{b}+\left[\bar{c}^{T}(j \omega I-\bar{A})^{-1} \bar{b}\right]^{*}$ $=-2 \bar{c}^{T}\left(\omega^{2} I+\bar{A}^{2}\right)^{-1} \bar{A} \bar{b}$.

Since $\bar{d}=-c^{T} A^{-1} b>0$, we can write

$$
\begin{aligned}
H( & \left.-j \omega^{-1}\right)+H\left(-j \omega^{-1}\right)^{*} \\
= & 2 \bar{d} \operatorname{det}\left[1-\frac{1}{\bar{d}} \bar{c}^{T}\left(\omega^{2} I+\bar{A}^{2}\right)^{-1} \bar{A} \bar{b}\right] \\
= & 2 \bar{d} \operatorname{det}\left[I-\frac{1}{\bar{d}}\left(\omega^{2} I+\bar{A}^{2}\right)^{-1} \bar{A} \bar{b} \bar{c}^{T}\right] \\
= & 2 \bar{d} \operatorname{det}\left[\left(\omega^{2} I+\bar{A}^{2}\right)^{-1}\right] \\
& \times \operatorname{det}\left[\omega^{2} I+\bar{A}^{2}-\frac{1}{\bar{d}} \bar{A} \bar{b} \bar{c}^{T}\right] .
\end{aligned}
$$

Thus

$$
H\left(-j \omega^{-1}\right)+H\left(-j \omega^{-1}\right)^{*}=\frac{2 \bar{d} \operatorname{det}\left[\omega^{2} I+M\right]}{\operatorname{det}\left[\omega^{2} I+\bar{A}^{2}\right]}
$$

where

$$
\begin{aligned}
M & :=\bar{A}\left(\bar{A}-\frac{1}{\bar{d}} \bar{b} \bar{c}^{T}\right) \\
& =A^{-1}\left(A^{-1}-\frac{A^{-1} b c^{T} A^{-1}}{c^{T} A^{-1} b}\right) .
\end{aligned}
$$

Since $A$ is Hurwitz, all the real eigenvalues of $\bar{A}^{2}=A^{-2}$ are positive which implies that $\operatorname{det}\left[\omega^{2} I+\bar{A}^{2}\right] \neq 0$ for all $\omega$. Noting that $\operatorname{det}\left[\omega^{2} I+\bar{A}^{2}\right]>0$ for $\omega$ sufficiently large, it follows from continuity arguments that $\operatorname{det}\left[\omega^{2} I+\bar{A}^{2}\right]>0$ for all $\omega$. Recalling that $\bar{d}>0$, it follows from identity (12) that conditions (9) and (10) on $H\left(-j \omega^{-1}\right)$ are respectively equivalent to

$$
\begin{aligned}
\operatorname{det}\left[\omega^{2} I+M\right] & >0 \quad \forall \quad \omega \in \mathbb{R}, \quad \omega \neq 0 \\
\lim _{\omega \rightarrow 0} \frac{1}{\omega^{2}} \operatorname{det}\left[\omega^{2} I+M\right] & >0 .
\end{aligned}
$$

Since $\operatorname{det}\left[\omega^{2} I+M\right]>0$ for large $\omega$, the above conditions are equivalent to

$$
\begin{aligned}
\operatorname{det}[\lambda I-M] & \neq 0 \quad \forall \quad \lambda \in \mathbb{R}, \quad \lambda<0 \\
\lim _{\lambda \rightarrow 0} \frac{1}{\lambda} \operatorname{det}[\lambda I-M] & \neq 0 .
\end{aligned}
$$

Condition (13) is equivalent to the requirement that $M$ has no negative real eigenvalues. Since $M b=0$ and $b \neq 0$, the matrix $M$ must have at least one zero eigenvalue; hence $\operatorname{det}[\lambda I-M]=\lambda q(\lambda)$ and all the other eigenvalues of $M$ are given by the roots of the polynomial $q$. Thus condition (14) is equivalent to $q(0) \neq 0$, that is, zero is not a root of $q$. Thus (14) is equivalent to the requirement that $M$ has only one eigenvalue at zero.

The equivalence between the first and third statement of the lemma follows from the SPR equivalence of $c^{T}\left(s I-A^{-1}\right)^{-1} b$ and $c^{T}(s I-$ $A)^{-1} b$ as stated in Lemma 2.2.

2) Comment 3: The literature contains spectral conditions for checking SPR of a strictly proper transfer function [10]; however, these conditions involve the eigenvalues of a $2 n \times 2 n$ Hamiltonian matrix. The conditions here involve a matrix of dimension $n \times n$.

\section{MAIN RESULT}

In everything that follows, $A$ is a real $n \times n$ matrix and $g$ and $h$ are real $n$-vectors. These results make use of the following observations.
A matrix $P=P^{T}>0$ is a strict Lyapunov matrix for $A$, that is

$$
A^{T} P+P A<0
$$

if and only if $P$ is a strict Lyapunov matrix for $A^{-1}$, that is

$$
A^{-T} P+P A^{-1}<0 \text {. }
$$

To see, this post- and pre-multiply the first inequality by $A^{-1}$ and its transpose.

In a similar fashion one can also show that $P$ is a (nonstrict) Lyapunov matrix for $A$, that is

$$
A^{T} P+P A \leq 0
$$

if and only if $P$ is a (nonstrict) Lyapunov matrix for $A^{-1}$, that is,

$$
P A^{-1}+A^{-T} P \leq 0 .
$$

The proof of the main result requires the following KYP lemma.

Lemma 3.1: [7]: Suppose $(A, b)$ is controllable and $(A, c)$ is observable. Then, the following statements are equivalent.

(i) The matrix $A$ is Hurwitz and the transfer function $H(s)=$ $c^{T}(s I-A)^{-1} b$ is SPR.

(ii) There exists a matrix $P=P^{T}>0$ that satisfies the constrained Lyapunov inequality

$$
\begin{aligned}
A^{T} P+P A & <0 \\
P b & =c .
\end{aligned}
$$

1) Comment 4: A discussion of strictly positive real transfer functions can be found in the book [14] by Narendra \& Taylor on frequency domain stability criteria. The assumption that $(A, c)$ is observable ensures that $P$ is positive definite [15].

Theorem 3.1 (Main Theorem): Suppose that $A$ is Hurwitz and all the eigenvalues of $A-g h^{T}$ have negative real part, except one, which is zero. Suppose also that $(A, g)$ is controllable and $(A, h)$ is observable. Then, there exists a matrix $P=P^{T}>0$ such that (15) and (16) hold if and only if the matrix product $A\left(A-g h^{T}\right)$ has no real negative eigenvalues and exactly one zero eigenvalue.

Proof: The proof consists of two parts. First we use an equivalence to show that the conditions on $A\left(A-g h^{T}\right)$ are sufficient for the existence of a Lyapunov matrix $P$ with the required properties. We then show that these conditions are also necessary.

Sufficiency: Let $b$ be a right eigenvector of $A-g h^{T}$ corresponding to the zero eigenvalue; then $b \neq 0$ and $A b=g h^{T} b=h^{T} b g$. Let $c=A^{-T} h$; then $c^{T} A b=h^{T} b$. Since $A$ is Hurwitz, we must have $h^{T} b \neq 0$, otherwise $A b=0$. Hence $c^{T} A b \neq 0$ and, without loss of generality, we assume that $b$ is chosen so that $c^{T} A b=-1$. In this case

$$
g=-A b \text { and } h^{T}=c^{T} A .
$$

Controllability of $(A, b)$ and observability of $(A, c)$ follow from controllability of $(A, g)$ and observability of $(A, h)$, respectively. Noting that

$$
A_{2}:=A-g h^{T}=A-\frac{A b c^{T} A}{c^{T} A b}
$$

it follows from Theorem 2.1 that the conditions on $A A_{2}$ imply that the transfer function $c^{T}(s I-A)^{-1} b$ is SPR. Consequently, it follows from Lemma 3.1 that there exists a matrix $P=P^{T}>0$ such that (15) and (16) hold. Pre- and post- multiplying inequality (15) by $A^{-T}$ and $A^{-1}$ shows that this inequality is equivalent to

$$
A^{-T} P+P A^{-1}<0
$$


Inequality (17) and (16) imply that

$$
\left[\begin{array}{cc}
A^{-T} P+P A^{-1} & P b-c \\
b^{T} P-c^{T} & 0
\end{array}\right] \leq 0
$$

that is

$$
\left[\begin{array}{ll}
A^{-1} & b \\
-c^{T} & 0
\end{array}\right]^{T}\left[\begin{array}{ll}
P & 0 \\
0 & 1
\end{array}\right]+\left[\begin{array}{ll}
P & 0 \\
0 & 1
\end{array}\right]\left[\begin{array}{ll}
A^{-1} & b \\
-c^{T} & 0
\end{array}\right] \leq 0 .
$$

Since $c^{T} A b=-1 \neq 0$

$$
\begin{aligned}
{\left[\begin{array}{cc}
A^{-1} & b \\
-c^{T} & 0
\end{array}\right]^{-1} } & =\left[\begin{array}{cc}
A-\frac{A b c^{T} A}{c^{T} A b} & -\frac{A b}{c^{T} A b} \\
\frac{c^{T} A}{c^{T} A b} & \frac{1}{c^{T} A b}
\end{array}\right] \\
& =\left[\begin{array}{cc}
A-g h^{T} & -g \\
-h^{T} & -1
\end{array}\right] .
\end{aligned}
$$

Post- and pre-multiplying inequality (18) by the above inverse and its transpose results in

$$
\left[\begin{array}{ll}
A^{-1} & b \\
-c^{T} & 0
\end{array}\right]^{-T}\left[\begin{array}{ll}
P & 0 \\
0 & 1
\end{array}\right]+\left[\begin{array}{ll}
P & 0 \\
0 & 1
\end{array}\right]\left[\begin{array}{ll}
A^{-1} & b \\
-c^{T} & 0
\end{array}\right]^{-1} \leq 0 .
$$

that is,

$$
\left[\begin{array}{cc}
\left(A-g h^{T}\right)^{T} P+P\left(A-g h^{T}\right) & -P g-h \\
-g^{T} P-h^{T} & -2
\end{array}\right] \leq 0 .
$$

It immediately follows that for the above inequality to hold, we must have

$$
\left(A-g h^{T}\right)^{T} P+P\left(A-g h^{T}\right) \leq 0 .
$$

Necessity: We first show that if there exists a matrix $P=P^{T}>0$ satisfying conditions (15)-(16), then $A A_{2}$ cannot have a negative real eigenvalue. Note that the conditions on $P$ are equivalent to

$$
\begin{array}{r}
A^{-T} P+P A^{-1}<0 \\
A_{2}^{T} P+P A_{2} \leq 0 .
\end{array}
$$

Hence, for any $\gamma>0$

$$
\left(A_{2}+\gamma A^{-1}\right)^{T} P+P\left(A_{2}+\gamma A^{-1}\right)<0 .
$$

Since $P=P^{T}>0$, this Lyapunov inequality implies that $A_{2}+$ $\gamma A^{-1}$ must be Hurwitz and hence, nonsingular. Thus, $A A_{2}+\gamma I$ is nonsingular for all $\gamma>0$. This means that $A A_{2}$ cannot have a negative real eigenvalue.

We now show that $A A_{2}$ cannot have a zero eigenvalue whose multiplicity is greater than one. To this end, introduce the matrix

$$
\tilde{A}(k)=A_{2}+k g h^{T} .
$$

Then $A=\tilde{A}$ (1) and inequalities (15)-(16) hold if and only if

$$
\begin{array}{r}
\tilde{A}(k)^{T} P+P \tilde{A}(k)<0 \\
A_{2}^{T} P+P A_{2} \leq 0
\end{array}
$$

hold for all $k$ sufficiently close to one. As we have seen above, this implies that $\tilde{A}(k) A_{2}$ cannot have negative real eigenvalues for all $k$ sufficiently close to one. We shall show that $A A_{2}$ having an eigenvalue at the origin whose multiplicity is greater than one contradicts this statement.

By assumption, $A_{2}$ has a single eigenvalue at zero; a corresponding eigenvector is the vector $b$. Clearly, $b$ is also an eigenvector corre- sponding to a zero eigenvalue of $\tilde{A}(k) A_{2}$. Now choose any nonsingular matrix $T$ whose first column is $b$. Then,

$$
T^{-1} \tilde{A}(k) A_{2} T=\left[\begin{array}{cc}
0 & * \\
0 & S+k r s^{T}
\end{array}\right]
$$

and the eigenvalues of $\tilde{A}(k) A_{2}$ consist of zero and the eigenvalues of $S+k r s^{T}$. Note that the matrix $S$ must be invertible since

$$
T^{-1} A_{2}^{2} T=T^{-1} \tilde{A}(0) A_{2} T=\left[\begin{array}{ll}
0 & * \\
0 & S
\end{array}\right]
$$

and $A_{2}^{2}$ has only a single eigenvalue at zero. Now suppose that $A A_{2}=$ $\tilde{A}(1) A_{2}$ has an eigenvalue at the origin whose multiplicity is greater than one. Then $S+r s^{T}$ must have a eigenvalue at zero; hence, det $[S+$ $\left.r s^{T}\right]=0$. Since $S$ is invertible

$$
\begin{aligned}
\operatorname{det}\left[S+k r s^{T}\right] & =\operatorname{det}[S] \operatorname{det}\left[I+k S^{-1} r s^{T}\right] \\
& =\operatorname{det}[S]\left(1+k s^{T} S^{-1} r\right)
\end{aligned}
$$

and we must have $1+s^{T} S^{-1} r=0$ which implies that $s^{T} S^{-1} r=-1$. Hence

$$
\operatorname{det}\left[S+k r s^{T}\right]=\operatorname{det}[S](1-k) .
$$

Suppose $\operatorname{det}[S]>0$. Then

$$
\operatorname{det}\left[S+k r s^{T}\right]<0
$$

for $k>1$. Since $\operatorname{det}\left[S+k r s^{T}\right]$ is the product of all the eigenvalues of $S+k r s^{T}$ and complex eigenvalues occur in complex conjugate pairs, $S+k r s^{T}$ must have at least one real negative eigenvalue when $k>1$. This yields the contradiction that $\tilde{A}(k) A_{2}$ has a negative real eigenvalue when $k>1$. The conclusion is the same for $\operatorname{det}[S]<0$.

2) Comment 5: Let $G(s)=h^{T}(s I-A)^{-1} g+1$. Then one can readily show that satisfaction of the Lyapunov inequalities (3) is also equivalent to the following frequency domain conditions.

$$
\begin{aligned}
G(j \omega)+G(j \omega)^{*} & >0 \text { for } \omega \neq 0 \\
\lim _{\omega \rightarrow 0} \frac{1}{\omega^{2}}\left[G(j \omega)+G(j \omega)^{*}\right] & >0 .
\end{aligned}
$$

One can view this as a KYP result for the systems under consideration.

To demonstrate this result, recall Lemma 2.3 and the definitions of $b$ and $c$ in the proof of Theorem 3.1 to obtain that

$$
G\left(s^{-1}\right)=c^{T}\left(s I-A^{-1}\right)^{-1} b .
$$

The proof of Theorem 3.1 and Lemma 2.2 tell us that satisfaction of the Lyapunov inequalities (3) is equivalent to the transfer function $c^{T}$ ( $s I-$ $\left.A^{-1}\right)^{-1} b$ being SPR. Using the fact that $c^{T} A b=-1$, the desired result now follows from Lemma 2.1.

\section{EXAMPLES}

In this section we present two examples to illustrate the main features of our result.

1) Example 1 (No Common Quadratic Lyapunov Function): Consider

$$
A_{1}=\left[\begin{array}{ccc}
0 & 1 & 0 \\
0 & 0 & 1 \\
-1 & -2 & -3
\end{array}\right], \quad A_{2}=\left[\begin{array}{ccc}
0 & 1 & 0 \\
0 & 0 & 1 \\
0 & -1 & -2
\end{array}\right]
$$

with $A_{2}=A_{1}-g h^{T}$ and $g^{T}=\left[\begin{array}{lll}0 & 0 & 1\end{array}\right]$ and $h^{T}=$ $\left[\begin{array}{lll}-1 & -1 & -1\end{array}\right]$. Note that $A_{1}$ is Hurwitz; whereas $A_{2}$ is singular with all its eigenvalues in the open left half of the complex plane, except one at the origin. Note also that $(A, g)$ and $(A, h)$ are 
controllable and observable, respectively. The eigenvalues of the matrix product $A_{1} A_{2}$ are $(0,0,3)$. Hence, from the results of our main theorem, there cannot exist a $P=P^{T}>0$ such that $A_{1}^{T} P+P A_{1}<0$ and $A_{2}^{T} P+P A_{2} \leq 0$.

2) Example 2 (Quadratic Stability): Consider

$$
A_{1}=\left[\begin{array}{ccc}
0 & 1 & 0 \\
0 & 0 & 1 \\
-.9 & -1.9 & -2.9
\end{array}\right], \quad A_{2}=\left[\begin{array}{ccc}
0 & 1 & 0 \\
0 & 0 & 1 \\
0 & -1 & -2
\end{array}\right]
$$

with $A_{2}=A_{1}-g h^{T}$ and $g^{T}=\left[\begin{array}{lll}0 & 0 & 1\end{array}\right]$ and $h^{T}=$ $\left[\begin{array}{lll}-.9 & -.9 & -.9\end{array}\right]$. Note that $A_{1}$ is Hurwitz; whereas $A_{2}$ is singular with all its eigenvalues in the open left half of the complex plane, except one at the origin. Note also that $(A, g)$ and $(A, h)$ are controllable and observable, respectively. The eigenvalues of the matrix product $A_{1} A_{2}$ are $(0,0.0349,2.8651)$. Hence, from the main theorem, there exist a $P=P^{T}>0$ such that $A_{1}^{T} P+P A_{1}<0$ and $A_{2}^{T} P+P A_{2} \leq 0$.

\section{CONCLUSION}

In this note, we have derived necessary and sufficient conditions for the existence of a common quadratic Lyapunov function for a pair of stable linear time-invariant systems whose system matrices are of the form $A, A-g h^{T}$, and where one of the matrices is singular. As a preliminary result, we obtained a spectral characterization of strictly positive real transfer functions which are strictly proper. Future work will involve extending our results to nonquadratic Lyapunov functions such as those which arise in the application of Popov's criterion.

\section{APPENDIX}

First Note: Here we show that satisfaction of the Lyapunov inequalities (3) imply that the matrix $A-g h^{T}$ can have at most one zero eigenvalue and cannot have a nonzero imaginary eigenvalue. Consider first nonzero imaginary eigenvalues. Suppose on the contrary that, $j \omega$ is a nonzero imaginary eigenvalue of $A-g h^{T}$ with eigenvector $v$ and let $Q_{2}=\left(A-g h^{T}\right)^{T} P+P\left(A-g h^{T}\right)$. Then

$$
\begin{aligned}
v^{\prime} Q_{2} v & =\left[\left(A-g h^{T}\right) v\right]^{\prime} P v+v^{\prime} P\left[\left(A-g h^{T}\right) v\right] \\
& =[j \omega v]^{\prime} P v+v^{\prime} P[j \omega v] \\
& =0 .
\end{aligned}
$$

Since $A-g h^{T}$ is real, $-j \omega$ is an eigenvalue of $A-g h^{T}$ with eigenvector $\bar{v}$; hence $\bar{v}^{\prime} Q_{2} \bar{v}=0$. The second inequality in (3) tells us that $Q_{2} \leq 0$; hence, the set of vectors for $x$ which $x^{\prime} Q_{2} x=0$ is the same as the null space of $Q_{2}$. Since the linearly independent vectors $v$ and $\bar{v}$ are in this null space, this space has dimension greater than one. Let $Q_{1}=A^{T} P+P A$. Then $Q_{1}=Q_{2}+P g h^{T}+h g^{T} P$ and when $x$ is in the null space of $h^{T}$, it should be clear that $x^{\prime} Q_{1} x=x^{\prime} Q_{2} x$. Since the dimension of the null space of $h^{T}$ is $n-1$ and the dimension of the null space of $Q_{2}$ is greater than one, these two spaces have a nonzero common element $x$. For this $x$, we have

$$
x^{\prime} Q_{1} x=x^{\prime} Q_{2} x=0 .
$$

and we obtain the contradiction that the first inequality in (3) does not hold. Hence $A-g h^{T}$ cannot have nonzero imaginary eigenvalues.

Now suppose that $A-g h^{T}$ has multiple eigenvalues at zero. It follows from the second inequality in (3) that the system $\dot{x}=\left(A-g h^{T}\right) x$ is stable; hence the algebraic multiplicity and geometric multiplicity of the zero eigenvalue are the same. This implies that there are at least two linearly independent eigenvectors for the zero eigenvalue. Proceeding now as in the case of the nonzero imaginary eigenvalues, one can obtain a contradiction. Hence, $A-g h^{T}$ cannot have a repeated eigenvalue at zero.

Second Note: Here we show that if $A-g h^{T}$ is marginally stable, $A$ is Hurwitz and the conditions on $A\left(A-g h^{T}\right)$ hold then, the matrix
$A-g h^{T}$ can only have one zero eigenvalue and cannot have a nonzero imaginary eigenvalue. Consider first nonzero imaginary eigenvalues. Suppose on the contrary that, $j \omega$ is a nonzero imaginary eigenvalue of $A-g h^{T}$ with eigenvector $v$. Then $-j \omega$ is a nonzero imaginary eigenvalue of $A-g h^{T}$ with eigenvector $\bar{v}$. From this, it follows that the linear independent vectors $v$ and $\bar{v}$ are eigenvectors of $\left(A-g h^{T}\right)^{2}$ with eigenvalue $-\omega^{2}$. This implies that the eigenspace of $\left(A-g h^{T}\right)^{2}$ associated with $-\omega^{2}$ is greater than one. Since the dimension of the null space of $h^{T}$ is $n-1$ and the dimension of the above eigenspace is greater than one, these two spaces have a nonzero common element $x$. For this $x$, we have

$$
A\left(A-g h^{T}\right) A x=A\left(A-g h^{T}\right)^{2} x=-\omega^{2} A x .
$$

Since $A$ is Hurwitx, $A x$ is nonzero and the above yields the contradiction that $A\left(A-g h^{T}\right)$ has a negative real eigenvalue. Hence $A-g h^{T}$ cannot have nonzero imaginary eigenvalues.

Now suppose that $A-g h^{T}$ has multiple eigenvalues at zero. Since the system $\dot{x}=\left(A-g h^{T}\right) x$ is marginally stable; the algebraic multiplicity and geometric multiplicity of the zero eigenvalue must be the same. This implies that there are at least two linearly independent eigenvectors for the zero eigenvalue of $A-g h^{T}$. These are also eigenvectors for the zero eigenvalue of $A\left(A-g h^{T}\right)$. This contradicts the fact that $A\left(A-g h^{T}\right)$ has a single eigenvalue at zero. Hence, $A-g h^{T}$ cannot have a repeated eigenvalue at zero.

\section{REFERENCES}

[1] M. V. Kothare and M. Morari, "Multiplier theory for stability analysis of anti-windup control systems," Automatica, vol. 35, pp. 917-928, 1999.

[2] B. G. Romanchuk and M. C. Smith, "Incremental gain analysis of piecewise linear systems and application to the antiwindup problem," Automatica, vol. 35, pp. 1275-1283, Jul. 1999.

[3] M. J. Gomes da Silva and S. Tarbouriech, "Antiwindup design with guaranteed regions of stability: An LMI-based approach," IEEE Trans. Autom. Control, vol. 50, no. 1, pp. 106-111, Jan. 2005.

[4] T. Loquen, S. Tarbouriech, and C. Prieur, "Stability analysis for reset systems with input saturation," in Proc. 46th IEEE Conf. Decision and Control, Dec. 2007, pp. 3272-3277.

[5] R. N. Shorten, K. S. Narendra, and O. Mason, "A result on common quadratic Lyapunov functions," IEEE Trans. Autom. Control, vol. 48, no. 1, pp. 110-113, Jan. 2003.

[6] R. N. Shorten and K. S. Narendra, "On common quadratic Lyapunov functions for pairs of stable LTI systems whose system matrices are in companion form," IEEE Trans. Autom. Control, vol. 48, no. 4, pp. 618-621, Apr. 2003.

[7] S. Boyd, L. El Ghaoui, E. Feron, and V. Balakrishnan, Linear Matrix Inequalities in System and Control Theory. Philadelphia, PA: SIAM, 1994.

[8] H. Khalil, Nonlinear Systems, 3rd ed. Englewood Cliffs, NJ: Prentice-Hall, 2002.

[9] R. Shorten and C. King, "Spectral conditions for positive realness of SISO systems," IEEE Trans. Autom. Control, vol. 49, no. 10, pp. 1875-1877, Oct. 2004.

[10] R. Shorten, P. Curran, K. Wulff, and E. Zeheb, "A note on spectral conditions for positive realness of transfer function matrices," IEEE Trans. Autom. Control, vol. 53, no. 6, pp. 1258-1261, Jun. 2008.

[11] Z. Bai and W. Freund, "Eigenvalue based characterization and test for positive realness of scalar transfer functions," IEEE Trans. Autom. Control, vol. 45, no. 12, pp. 2396-2402, Dec. 2000.

[12] G. Muscato, G. Nunnari, and L. Fortuna, "Singular perturbation approximation of bounded real and positive real transfer matrices," in Proc. ACC, Baltimore, MD, 1994, pp. 3416-3420.

[13] R. E. Kalman, "Lyapunov functions for the problem of Lur'e in automatic control," Proc. National Academy of Sciences, vol. 49, no. 2, pp. 201-205, 1963.

[14] K. Narendra and J. Taylor, Frequency Domain Criteria for Absolute Stability. New York: Academic, 1973.

[15] A. Acikmese and M. Corless, "Stability analysis with quadratic Lyapunov functions: Some necessary and sufficient multiplier conditions," Syst. Control Lett., vol. 57, pp. 78-94, 2008. 